\title{
THE APPLICATION OF AN AUTOMATED OXYGEN CONCENTRATION CONTROL AND MEASUREMENT SYSTEM TO A MINIATURIZED ENERGY CONSUMPTION MEASUREMENT SYSTEM USING RESISTIVE-TYPE OXYGEN GAS SENSORS
}

\author{
Chien-Chung Wu ${ }^{1}$, Gwo-Bin LeE ${ }^{2}$, Ching-Hsing Luo ${ }^{1}$ \\ ${ }^{1}$ Department of Electrical Engineering \\ ${ }^{2}$ Department of Engineering Science \\ National Cheng Kung University, Tainan, Taiwan
}

\begin{abstract}
Although the conventional indirect calorimeter is a valuable tool, its size and expense prohibits its widespread use in hospitals. Furthermore, its flow-through measurement technique dilutes the respiratory variations, and hence some form of high-precision detection instrumentation is required. These limitations may be overcome by combining MEMS with CMOS circuit design technology to develop an innovative SOC biochip as the basis of a miniaturized energy consumption measurement system. In order to measure the characteristics of the oxygen sensors which form one part of this system, this study develops an automated oxygen concentration control and measurement system. This system can simulate the miniscule respiratory variations of a premature infant and can subsequently establish a suitable oxygen concentration environment to ensure the infant's well being. The proposed system is capable of establishing environments with oxygen concentrations ranging from $5 \%$ to $100 \%$, and can control the oxygen concentration to a resolution of $0.006 \%$. The minimum time required to increase the oxygen concentration from $21 \%$ to $100 \%$ is approximately 5.6 seconds. The proposed system can also automatically measure the properties of the oxygen sensors, including their resistance characteristics at different oxygen concentrations, the relationship between their sensitivity and the oxygen concentration, and the influence of working temperature and humidity upon their sensitivity. The necessary measurement data is acquired locally and can then be transmitted to a remote $P C$ via the Internet.
\end{abstract}

Biomed Eng Appl Basis Comm, 2004 (February); 16: 22-31.

Keywords: Automated oxygen concentration control and measurement system, miniaturized energy consumption measurement system, Indirect calorimeter, Remote control system, Remote measurement system.

Received: Dec 2, 2003; Accepted: Dec 25, 2003

Correspondence: Ching-Hsing Luo, Professor

Department of Electrical Engineering, National Cheng

Kung University, Tainan, Taiwan 701

E-mail: robinluo@mail.ncku.edu.tw

\section{INTRODUCTION}

Due to high mental pressure, advanced age pregnancies, and a generally more stressful environment, the population of premature infants has been increasing recently. Since the organs of these 
infants tend not to be well formed, it is necessary to develop advanced instrumentation to nurse then to full health. These infants require an advanced growth formula which is very different from that received by their full term counterparts. Noninvasive methods of monitoring the health conditions of such infants are generally preferred. A previous study has reported that the metabolic rate is a valuable indicator of the growth status of premature babies [1]. Currently, one of the most frequently adopted metabolic rate measurement methods is the so-called Respiratory Quotient (RQ) value, which is defined as the ratio of carbon-dioxide generation to oxygen consumption. Basically, the RQ value represents the oxidized substrates within a body, and as such, provides clinical doctors with an important metabolic indicator. In general, the $\mathrm{RQ}$ value is equal to one for carbohydrates $\left(\mathrm{CO}_{2}: \mathrm{O}_{2}=1\right), 0.8$ for proteins, and 0.7 for fats [2]. A reduction in the RQ value indicates a decreasing rate of carbohydrate metabolism and a shift towards alternative substrates such as proteins and particularly, fats. Previous studies have suggested that the quantity of proteins deposited is closely related to the protein intake, while the quantity of fat deposited is closely related to the energy intake [3]. Therefore, the RQ value provides a valuable indicator of a premature infant's energy intake.

Traditionally, RQ values have been determined by means of a noninvasive method which employs an indirect calorimeter in a flow-through technique [4-6]. In this technique, the respective concentrations of the oxygen and carbon dioxide gases are measured during inspiration and expiration. However, any minor variations in the oxygen concentration tend to be diluted by the flow-through air, and hence it is generally necessary to employ some form of highprecision gas analyzer for oxygen detection purposes [4-6]. Furthermore, the indirect calorimeter is bulky and rather expensive, and therefore tends not to be used on a routine basis in practical clinical applications or in homecare situations. A novel solution has been proposed to overcome these limitations in which MEMS and CMOS circuit design technologies are combined to develop an innovative SOC biochip as the core of a miniaturized energy consumption measurement system [7-10]. However, further work is required to simulate the variation in the oxygen concentration of an infant's respiration, to establish a suitable oxygen environment to ensure the infant's recovery, and to measure the characteristics of the oxygen sensors employed within these measurement systems.

Since the aim of the miniaturized energy consumption measurement system is to monitor the energy consumption of a premature infant (i.e. to monitor the carbon-dioxide generation and the oxygen consumption), developing the oxygen control system involves three basic considerations, namely the actual oxygen environments employed in a clinical setting to promote the infant's well-being, the miniscule variations in oxygen content during the infant's respiration, and the infant's respiratory rate. It has been reported that the oxygen concentration of the inhaled gas is $20.84 \%$ for a full term newborn infant when the supplied gas is room air, and that the oxygen concentration of the corresponding exhaled gas ranges from $15.7 \%$ to $20.84 \%$ [11-12]. However, NRDS is often observed in premature infants because their pulmonary alveolus tends not to be fully developed. Therefore, in the clinical environment, it is frequently necessary to supply the infant with an oxygen concentration exceeding $21 \%$. Consequently, the present system is designed with the capability to supply oxygen concentrations in the range of $5 \%$ to $100 \%$. Secondly, developing the oxygen control system requires a full understanding of the oxygen volume consumption during a premature infant's respiratory cycle. Previously, it has been reported that full term newborn infants consume an oxygen volume of approximately $6.66 \pm 1.244(\mathrm{ml} / \mathrm{kg} / \mathrm{min})$ during the period of 6 hours from birth and prior to milk feed-in. Meanwhile, the generated carbon dioxide volume during the same period is approximately $6.305 \pm 1.259$ $(\mathrm{ml} / \mathrm{kg} / \mathrm{min})$. Furthermore, newborn infants within 30 hours from birth and prior to the start of milk feed-in consume an oxygen volume of approximately 7.422 \pm $1.241(\mathrm{ml} / \mathrm{kg} / \mathrm{min})$, and generate a carbon dioxide volume of approximately $6.532 \pm 1.091(\mathrm{ml} / \mathrm{kg} / \mathrm{min})$ [3]. Finally, it has been reported that the respiratory rate of newborn infants is approximately 40-60 breaths $/ \min [3,12]$.

The current authors, Wu et al. designed and fabricated the oxygen sensor illustrated schematically in Figure 1. The sensor measures $500 \mu \mathrm{m} \times 500 \mu \mathrm{m}$, and incorporates a pair of sensing electrodes connected to a sensing film and a pair of heating electrodes attached to a heater located beneath the sensing film. The main structure of the sensor is composed of a lowstress nitride suspended on a silicon substrate. The principal aim of this structure is to reduce the heat loss, and hence to minimize the power consumption of the oxygen sensor [10].

The sensitivity of the oxygen sensor is related to the working temperature and the relative humidity $[10$, 13-19]. Furthermore, it has been reported that the rate of resistance increase of the sensing film is proportional to the oxygen concentration [10]. This implies that the resistance of the tin-oxide based oxygen sensor is not constant in fixed oxygen concentrations. Thus a system capable of constant monitoring of oxygen concentration is inevitable.

It is clearly necessary to consider the oxygen sensor properties which are to be measured in the 


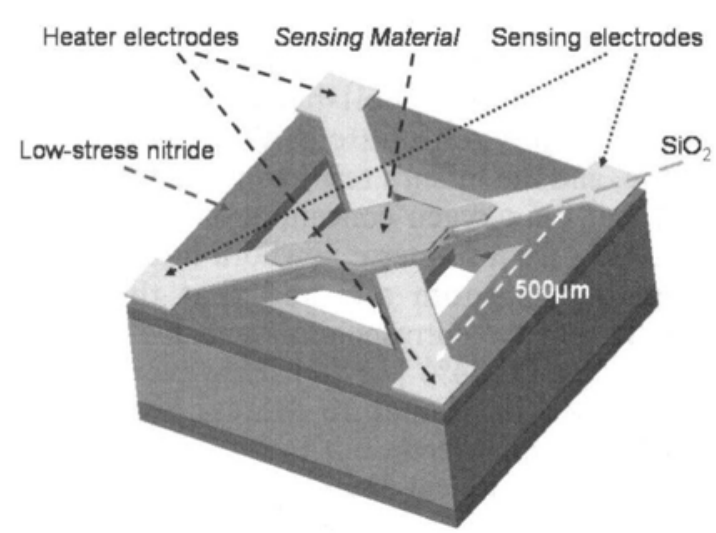

Fig. 1. Schematic representation of oxygen gas sensor. [10]

measurement system This study employs an oxygen concentration control and measurement system to perform the simultaneous measurement of several properties of the oxygen sensor and its environment, namely, the sensor resistance, the working temperature, the relative humidity, and the oxygen concentration. The properties of the oxygen sensor are dependent on the measurement time, and hence it is desirable to adopt an extended measurement period. To alleviate the intensive human interference involved in the measurement process, the proposed measurement system is designed to operate automatically under the control of remote PCs acting through an Internet interface. Figure 2 provides a block diagram of the automated oxygen concentration control and measurement system.

\section{METHOD}

The proposed system involves a hardware implementation and a software implementation.

\subsection{Hardware Implementation.}

The system (see Fig. 2) comprises two major hardware components: (1) an oxygen control component incorporating four mass flow controllers, a flow control unit, a hood, pure nitrogen gas, a hospital supplemental system (i.e. pure oxygen and room air), and a hospital vacuum system, and (2) a data acquisition component, which comprises a DC programmable power supply with RS-232C (LPS 305/R/CE, MOTECH Industries Inc.), two multifunction digital meters with RS-232C (TES 2801, Test Instruments Inc.), a high-precision ( $0.1 \%$ ) wellcalibrated differential paramagnetic oxygen gas analyzer (Magnos 16, Hartmann \& Braun Inc.), a humidity sensor (Rhu207, Homes), an Ethernet Hub, and a control PC (Pentium II 233,128M RAM) with two network interface cards and a high-speed serial interface card (W-2100-9835-2S1P, WINIC Inc.).

In order to simulate the variations in oxygen concentrations during the premature infant's respiratory cycle, the present system adopts the use of three types of supplied gas rather than the conventional two types [6]. Specifically, this study considers oxygen concentrations of room air, pure oxygen and nitrogen of $20.84 \%, 100 \%$ and $0 \%$, respectively. Using these values, the proposed oxygen control equation can be expressed as follows:

$$
\operatorname{Oxygen}(\text { Vol\% })=\frac{B_{\text {flow }} \times 20.84 \%+C_{\text {flow }} \times 100 \%+D_{\text {flow }} \times 0 \%}{B_{\text {flow }}+C_{\text {flow }}+D_{\text {flow }}}
$$

where $A_{\text {flow }}, B_{\text {flow }}, C_{\text {flow }}$ and $D_{\text {flow }}$ denote the gas flows through mass flow controllers $\mathrm{A}, \mathrm{B}, \mathrm{C}$ and $\mathrm{D}$, respectively.

To establish a non-interference measurement environment, the total flow into the hood (i.e. the sum of $B_{\text {flow }}, C_{\text {flow }}$ and $D_{\text {flow }}$ ) must be controlled such that it is greater than the flow out of the hood (i.e. $A_{\text {flow }}$ ). Hence, it is vital to develop a single dedicated unit to control and coordinate all of the individual mass flow controllers in the system. This study employs a control flow unit comprising a single 89C51 chip to control four 8-bit DACs, which then control the individual mass flow controllers (flow $\max =10 \mathrm{l} / \mathrm{min}$ ) by varying the supply voltage from 0 to $5 \mathrm{~V}$. With this approach, the four mass flow controllers can be remotely controlled via an RS-232C interface.

The miniature dimensions of the oxygen sensor employed in the energy consumption system prohibit the direct measurement of the working temperature. However, Wu et al. have reported that the working

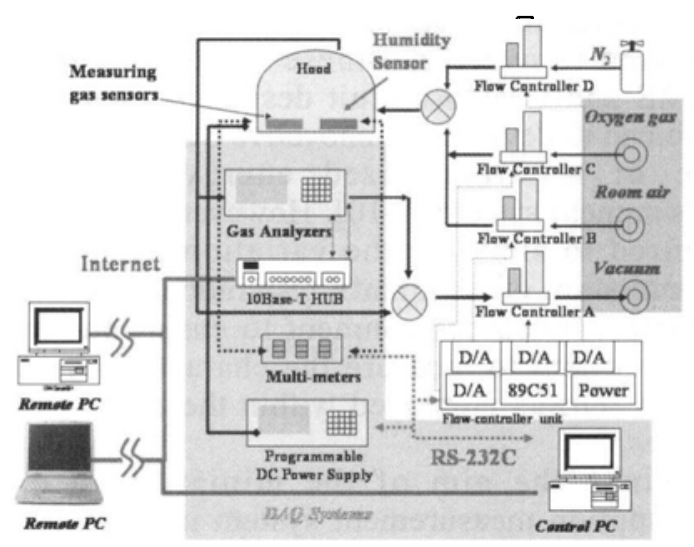

Fig. 2. Schematic block diagram of automated oxygen control and measurement system. 
temperature of an oxygen sensor is linearly related to the power consumption of its micro-heater [10]. Consequently, the working temperature can be calculated by monitoring the supplied voltage and current to the micro-heater.

In the proposed system, the data acquisition component employs a control PC to collect all of the measurement data. The output from the high-precision well-calibrated differential paramagnetic oxygen gas analyzer is collected to the control PC via Ethernet connection. The voltage and current are measured using a multi-function digital meter with an RS-232C function. Meanwhile, the relative humidity is monitored by measuring the output voltage of the humidity sensor located in the hood, and the resistance of the oxygen sensor and relative humidity are measured using additional multi-function digital meters. The voltage, current, resistance of oxygen sensor and relative humidity data are transferred to the control PC via RS-232C interfaces.

Unfortunately, even using the automatic measurement system described above, a precise measurement of the oxygen sensor characteristics is only possible if an extended measurement period is allowed. Therefore, the control PC is connected to the Internet such that the system may be monitored remotely without the need for constant physical supervision.

\subsection{Software Implementation.}

This system comprises three major software components: (1) an oxygen control program written in Microsoft Visual Basic 6.0, which is divided into three different functional sub-programs, i.e. a gas calibration program, an RS-232C interface, and an oxygen concentration control program, (2) a data acquisition program, which is also written in Microsoft Visual Basic 6.0. It is also composed of a control program with a programmable DC power supply, a data collection program interfacing with a multi-function digital meter, and a communication program to interface the oxygen gas analyzer and the file management system program, (3) an Internet remote control program using the pcAnywhere 9.2 remote control system designed by Symantec Corporation.

Since the oxygen concentration of the actual supply gas system used in a hospital environment does not have a constant value. For example, the concentration may actually be $98 \%$, etc. ), the proposed system incorporates a gas calibration program to calibrate the three supplied gas sources, i.e. pure oxygen $(100 \%$ oxygen), room air $(20.84 \%$ oxygen) and nitrogen ( $0 \%$ oxygen). In order to improve the accuracy of the oxygen concentration setting, the gas calibration procedure is performed prior to executing the oxygen control program. During gas calibration, mass flow controller $\mathrm{C}$ is opened initially; while the other mass flow controllers remain closed. The oxygen concentration is allowed to stabilize and then its value is recorded and subsequently applied in Equation (1). Subsequently, a similar procedure is adopted to calibrate the room air and nitrogen concentrations.

The individual mass flow controllers (A, B, C, and D) are controlled by the control PC, which sends appropriate commands to the overall flow control unit through the RS-232C interface port. The 89C51 chip then translates the received flow commands into the corresponding analogue voltages and transmits this voltage to the indicated mass flow controller via the appropriate DAC. The communication protocol between the control PC and the flow control unit is defined as follows: a 9600 BPS transmission and receiving speed, an 8-bit data length, a non-parity check bit, and a one stop bit.

The user-friendly interface of the oxygen control program enables the desired oxygen concentration recipes to be specified progressively by the user by means of pop-up menus. Figure 3 presents a schematic view of the structured oxygen control program. The complete oxygen concentration supply plan consists of a series of individual programs, each of which comprises a number of recipes specifying details of the required oxygen concentration values and their corresponding durations. Figure 4(a) provides an example of a typical oxygen concentration supply plan. It can be seen that the execution plans comprise two

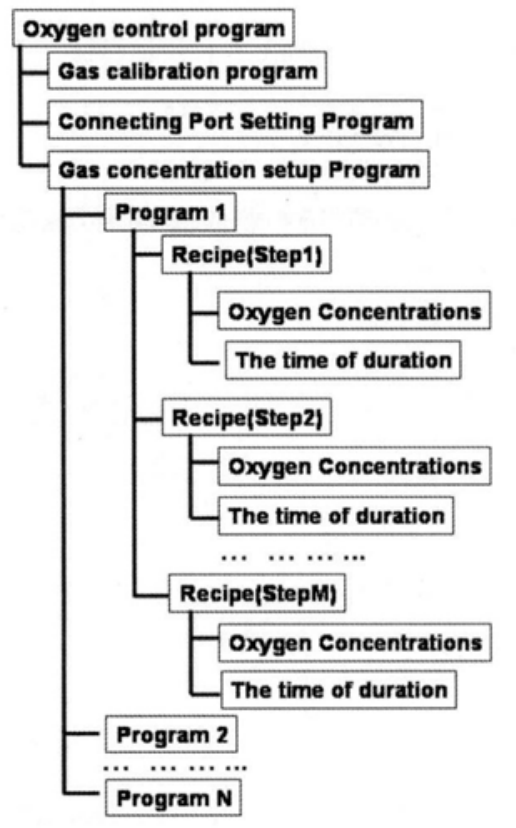

Fig. 3. Schematic structural diagram of oxygen control program. 
ordered programs, namely Program 1 and Program 2. Program 1 comprises four structured recipes, i.e. Recipe 1 (21\%, 1 min), Recipe 2 (30\%, $1 \mathrm{~min})$, Recipe 3 (21\%, $1 \mathrm{~min})$, and Recipe 4 (40\%, $1 \mathrm{~min})$. Program 2 also comprises four structured recipes, namely Recipe 1 (21\%, lmin), Recipe $2(30 \%, 1 \mathrm{~min})$, Recipe $3(40 \%$, $1 \mathrm{~min})$, and Recipe 4 (21\%, $1 \mathrm{~min})$. Figure 4(b) presents the screen provided to the user to specify the desired oxygen control program.

The power consumption of the micro-heater can be programmed by controlling the voltage and current supplied to the micro-heater via the data acquisition program. Figure 5 shows that the supply voltage was varied between $24 \mathrm{~V}$ and $28 \mathrm{~V}(+1$ volts/step, 5-minute wait/step) and that the data acquisition program was remotely controlled using the installed Symantec pcAnywhere software. Furthermore, the voltage and current supplied to the micro-heater, and the resistance of the oxygen sensor was recorded every 5 seconds during the present experiments.

\section{RESULTS AND DISCUSSION}

From Equation (1), it is clear that the same oxygen concentration can be obtained by using many different combinations of individual flow controller flow rates. In order to determine the relationship

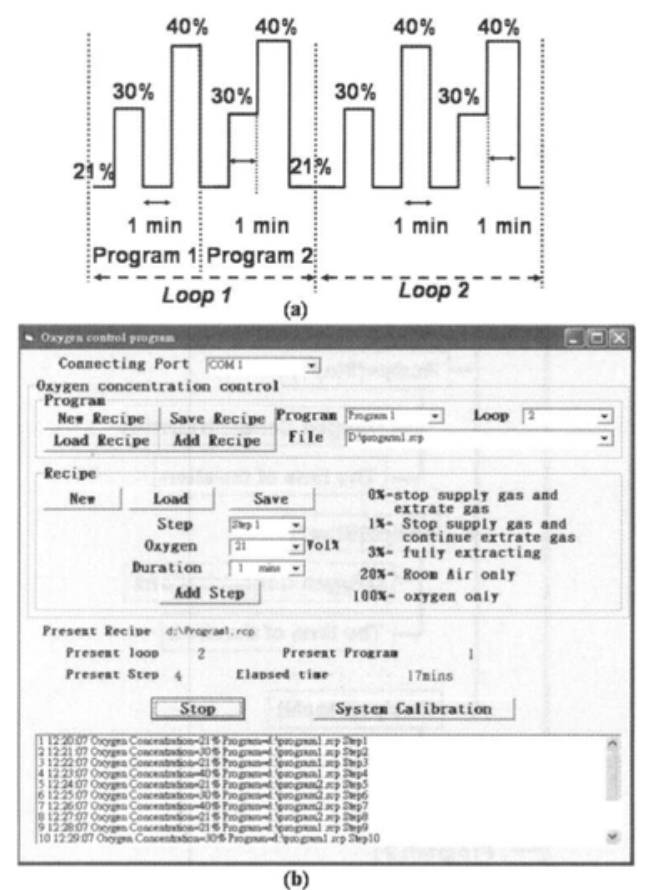

Fig. 4. (a) Schematic representation of oxygen supply sequences, (b) User input screen for oxygen control program specification. between the supply flow rate and the oxygen mixing ability, three recipes were specified, i.e. $20 \%(0.5 \mathrm{~min})$, $100 \%(0.5 \mathrm{~min})$, and $20 \%(0.5 \mathrm{~min})$. Note that initially the supply gas was room air ( $20 \%$ oxygen) supplied at $200 \mathrm{ml} / \mathrm{min}$. After 30 seconds, the supply gas was switched to pure oxygen (100\% oxygen) at a flow rate of $200 \mathrm{ml} / \mathrm{min}$, and maintained for a further 30 seconds, and then the supply gas was switched to room air to purge the chamber for a further 30 seconds. This complete cycle was then repeated, with the supply rate increasing by $100 \mathrm{ml} / \mathrm{min}$ each time. Afterwards, the same experiment then repeated, with the extracting flow rate was varied between $0 \mathrm{l} / \mathrm{min}$ and $5 \mathrm{l} / \mathrm{min}(+1$ 1/step). Figure 6 reveals that the oxygen mixing ability stabilizes for supply gas flow rates greater than 1100 $\mathrm{ml} / \mathrm{min}$. Furthermore, it can be seen that varying the extraction flow rate between $0 \mathrm{ml} / \mathrm{min}$ and 5000 $\mathrm{ml} / \mathrm{min}$ has insignificant influence upon the oxygen mixing ability.

The oxygen mixing time is also an important issue. In order to establish the relationship between the

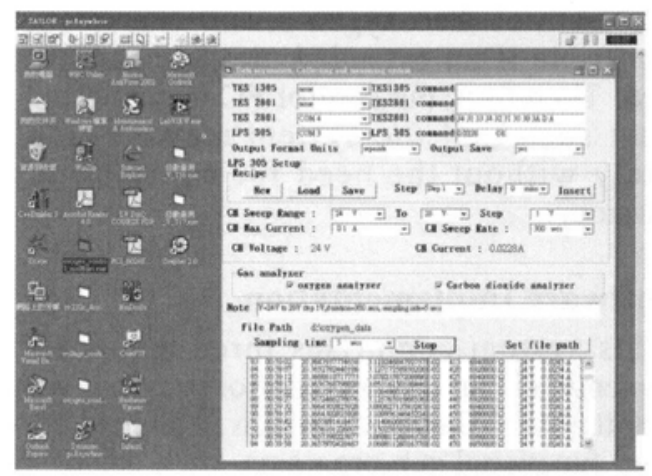

Fig. 5. User input screen for remotely controlling the data acquisition program by pcAnywhere 9.2 remote control program.

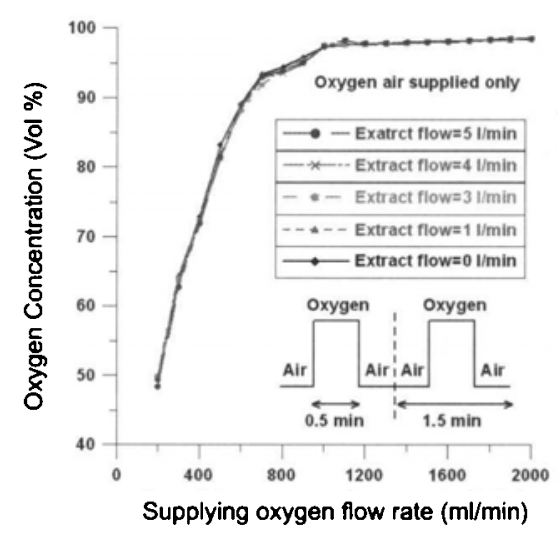

Fig. 6. Relationship between measured oxygen concentration and supply oxygen flow rate. 
supply flow rate and the required oxygen mixing time, the three recipes were specified as $20 \%(1 \mathrm{~min}), 100 \%$ $(0.5 \mathrm{~min})$, and $20 \%(0.5 \mathrm{~min})$. The extraction flow rate was fixed, and the oxygen supply flow rate was varied between $2000 \mathrm{ml} / \mathrm{min}$ and $10000 \mathrm{ml} / \mathrm{min}(+1000$ $\mathrm{ml} / \mathrm{step}, 30$-second wait/step). Figure 7 indicates that the oxygen mixing time reduces as the supply flow rate increases. The shortest time required to increase the oxygen concentration from $21 \%$ to $100 \%$ is approximately 5.6 seconds when the oxygen supply flow rate was $10000 \mathrm{ml} / \mathrm{min}$.

As mentioned previously, the respiration of premature infants in an NICU may demonstrate oxygen concentrations in the range of $15 \%$ to $100 \%$. Accordingly, in order to verify the accuracy of the proposed oxygen control program, the oxygen concentration was varied between $5 \%$ and $100 \%(+10$ $\% /$ step, 20-second wait/step) for a constant supply flow rate of $9000 \mathrm{ml} / \mathrm{sec}$ and an extraction flow rate of $2000 \mathrm{ml} / \mathrm{sec}$. Meanwhile, the sampling resolution of the data acquisition program was set at 2 points $/ \mathrm{sec}$. Figure 8 plots the relationship between the measured oxygen concentrations (solid line) and the specified concentrations (dotted line). It can be seen that there is a good agreement between the two sets of data.

After executing the calibration program, it is determined that the oxygen concentrations of room air, pure oxygen and nitrogen are $20.526 \%, 98.11 \%$ and $0.02 \%$, respectively. The gas supply system incorporates three 8-bit DACs in the flow control unit to control the individual mass flow controllers. Hence, a total of $16,777,216$ oxygen gas combinations can be generated. Two experiments are performed to explore the difference in the oxygen gas concentration control

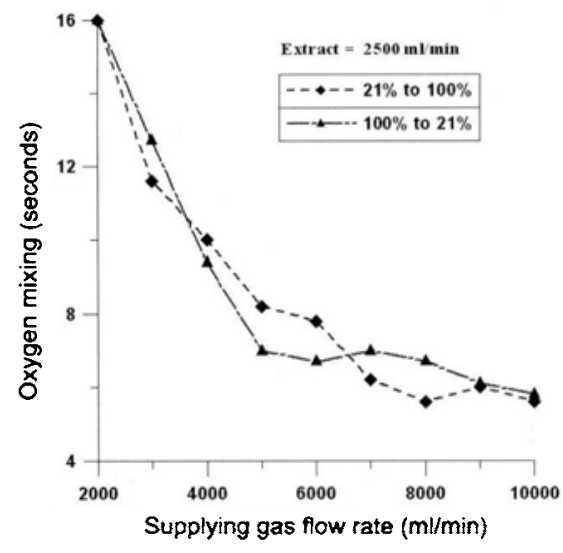

Fig. 7. Relationship between oxygen mixing time and supply gas flow rate.

resolution when three supply gases are employed rather than two. In the proposed system, the inputs of the 8-bit DACs range from 0 to 255 and are mapped to corresponding mass flow controller flow rates of 0 $\mathrm{ml} / \mathrm{min}$ to $10000 \mathrm{ml} / \mathrm{min}$. Table I considers the case where two gas sources are employed to generate oxygen concentrations in the range of $20.8 \%$ to $20.84 \%$. It is noted that a two-minute setting time is allowed before measurements are conducted. It can be seen that this concentration range is generated using only nine combinations of oxygen and room air. However, as shown in Table II, which presents the corresponding results for a three-gas supply, 9,830 different combinations of oxygen, room air and nitrogen are involved in obtaining oxygen concentrations in the same range. A process of

Table I. Oxygen concentrations ranging from $20.8 \%$ to $20.84 \%$ obtained using two gas sources, i.e. oxygen $(\mathbf{9 8 . 1 1 \%}$ ) and room air $(\mathbf{2 0 . 5 2 6 \% )}$. Note that the oxygen values were measured after 2 minutes of setting time.

\begin{tabular}{|l|c|c|c|c|}
\hline No. & Oxygen & Room Air & Simulation & Measured \\
\hline & $98.11 \%$ & $20.526 \%$ & $\%$ & $\%$ \\
\hline 1 & 1 & 255 & 20.829 & $20.830 \pm 0.020$ \\
\hline 2 & 1 & 254 & 20.830 & $20.830 \pm 0.015$ \\
\hline 3 & 1 & 253 & 20.831 & $20.830 \pm 0.020$ \\
\hline 4 & 1 & 252 & 20.833 & $20.830 \pm 0.012$ \\
\hline 5 & 1 & 251 & 20.834 & $20.830 \pm 0.010$ \\
\hline 6 & 1 & 250 & 20.835 & $20.840 \pm 0.010$ \\
\hline 7 & 1 & 249 & 20.836 & $20.840 \pm 0.020$ \\
\hline 8 & 1 & 248 & 20.838 & $20.840 \pm 0.020$ \\
\hline 9 & 1 & 247 & 20.839 & $20.840 \pm 0.021$ \\
\hline
\end{tabular}




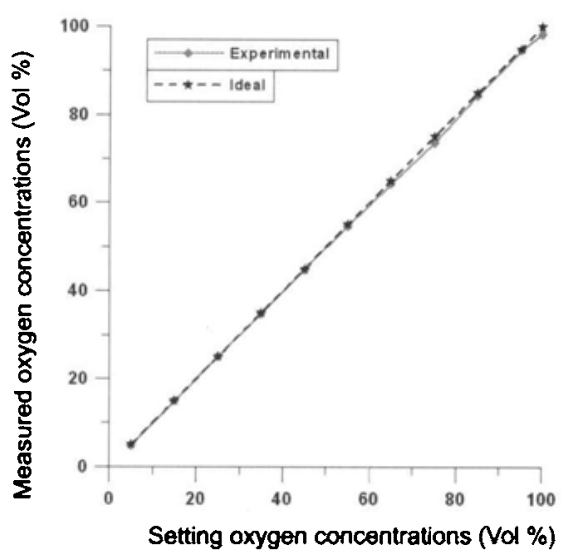

Fig 8. Relationship between measured oxygen concentration and specified oxygen concentrations in the range $5 \%$ to $100 \%$.

numerical analysis indicates that the control resolution of the oxygen concentration can theoretically be improved from $0.01 \%$ to $0.0001 \%$. However, in the present system, the measured improvement in resolution is actually from $0.042 \%$ to $0.006 \%$.

The proposed oxygen control program was also employed to simulate the respiratory rates and miniscule oxygen concentration variations of a premature infant's respiration by means of appropriate oxygen recipe programs which rapidly switched the flow rate and ratio of the supply gases to mimic a typical respiratory rate of 56 breaths per minute and oxygen concentrations ranging from $15.8 \%$ to $21.2 \%$. The corresponding simulation results are presented in Figure 9. The results confirm viability of the proposed system to simulate the miniscule variations in oxygen content during the infant's respiration, and the infant's respiratory rate.

Finally, in order to verify the performance of the complete system, an experimental plan was designed and executed for a complex oxygen concentration plan of extended duration. Figure 10 reveals the corresponding actual measurement results obtained from the micromachined oxygen sensors. During the experiment, the oxygen concentrations were varied between $21 \%$ and $40 \%(+10 \% /$ step, 765 -minute wait/step, 20-minute stop/step). In order to test the performance of the sensor under heating and cooling conditions, the DC programmable power supply voltage was initially varied between $1 \mathrm{~V}$ and $15 \mathrm{~V}(+1$ volts/step, 20-minute wait/step, 5-minute stop/step), and then subsequently varied between $15 \mathrm{~V}$ and $1 \mathrm{~V}(-1$ volts/step, 20-minute wait/step, 5-minute stop/step). The sampling rate of the data acquisition program was one sample every 5 seconds throughout, and the relative humidity was fixed at $20 \%$. The oxygen sensor corresponds well with the variation of the supplied oxygen concentrations. The results confirm the

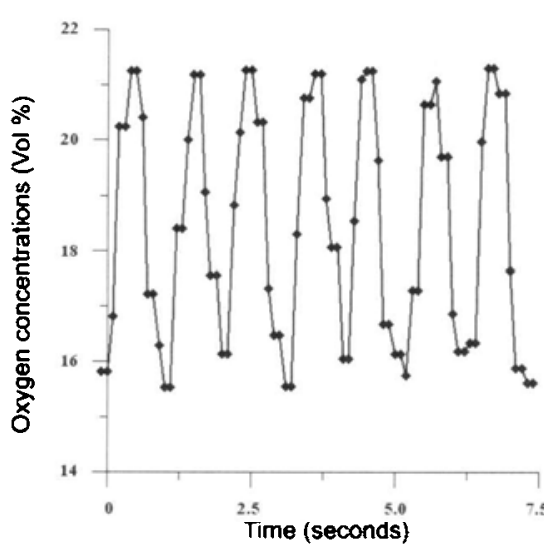

Fig. 9. Simulation of respiratory cycle of premature infant. Note that the sampling rate of the data acquisition program is one sample per 0.1 seconds.

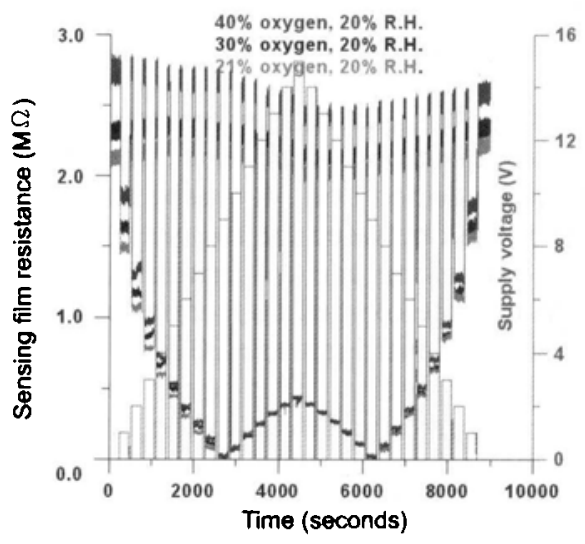

Figure 10. Actual measurement results from the micromachined oxygen sensors. The oxygen concentrations were varied between $21 \%$ and $\mathbf{4 0 \%}$ $(+10 \% /$ step, $765-$ minute wait/step, 20 -minute stop/step). The DC programmable power supply voltage was initially varied between $1 \mathrm{~V}$ and $15 \mathrm{~V}(+1$ volts/step, 20-minute wait/step, 5-minute stop/step), and then subsequently varied between $15 \mathrm{~V}$ and $1 \mathrm{~V}$ (-1 volts/step, 20-minute wait/step, 5-minute stop/step). The sampling rate of the data acquisition program was one sample per 5 seconds. The relative humidity was fixed at $20 \%$.

viability of the proposed system over an extended measurement period.

\section{CONCLUSIONS}

This paper has presented an automated oxygen concentration control and measurement system suitable 
Table II. Oxygen concentration ranging from $20.8 \%$ to $20.84 \%$ obtained using three gas sources, i.e. oxygen $(98.11 \%)$, room air $(20.526 \%)$ and nitrogen $(0.02 \%)$. Note that the oxygen values were measured after 2 minutes of setting time.

\begin{tabular}{|l|c|c|c|c|c|}
\hline No. & Oxygen & Room Air & Nitrogen & Simulation & Measured \\
\hline & $98.11 \%$ & $20.526 \%$ & $0.02 \%$ & $\%$ & $\%$ \\
\hline 1 & 42 & 247 & 153 & 20.80000 & $20.80000 \pm 0.003$ \\
\hline 2 & 51 & 132 & 188 & 20.80001 & $20.80001 \pm 0.002$ \\
\hline 3 & 60 & 17 & 223 & 20.80001 & $20.80001 \pm 0.002$ \\
\hline 4 & 14 & 234 & 49 & 20.80001 & $20.80001 \pm 0.001$ \\
\hline 5 & 23 & 119 & 84 & 20.80002 & $20.80002 \pm 0.002$ \\
\hline 6 & 46 & 238 & 168 & 20.80002 & $20.80002 \pm 0.002$ \\
\hline 7 & 55 & 123 & 203 & 20.80002 & $20.80002 \pm 0.003$ \\
\hline$\ldots \ldots$. & $\ldots \ldots \ldots$ & $\ldots \ldots$. & $\ldots \ldots$. & $\ldots \ldots \ldots \ldots$ & $\ldots \ldots \ldots \ldots \ldots \ldots \ldots .$. \\
\hline 9786 & 62 & 7 & 230 & 20.83981 & $20.83981 \pm 0.002$ \\
\hline 9787 & 62 & 206 & 227 & 20.83983 & $20.83983 \pm 0.002$ \\
\hline 9788 & 46 & 247 & 167 & 20.83983 & $20.83983 \pm 0.001$ \\
\hline 9789 & 55 & 141 & 202 & 20.83984 & $20.83984 \pm 0.002$ \\
\hline 9790 & 39 & 182 & 142 & 20.83984 & $20.83984 \pm 0.001$ \\
\hline 9791 & 23 & 223 & 82 & 20.83984 & $20.83984 \pm 0.002$ \\
\hline$\ldots \ldots .$. & $\ldots \ldots \ldots$ & $\ldots \ldots \ldots$ & $\ldots \ldots \ldots$ & $\ldots \ldots \ldots \ldots \ldots$ & $\ldots \ldots \ldots \ldots \ldots \ldots \ldots \ldots \ldots$ \\
\hline 9824 & 11 & 121 & 39 & 20.83998 & $20.83998 \pm 0.002$ \\
\hline 9825 & 22 & 242 & 78 & 20.83998 & $20.83998 \pm 0.001$ \\
\hline 9826 & 63 & 54 & 233 & 20.83998 & $20.83998 \pm 0.002$ \\
\hline 9827 & 40 & 229 & 145 & 20.83999 & $20.83999 \pm 0.003$ \\
\hline 9828 & 29 & 108 & 106 & 20.83999 & $20.83999 \pm 0.001$ \\
\hline 9829 & 58 & 216 & 212 & 20.83999 & $20.83999 \pm 0.001$ \\
\hline 9830 & 47 & 95 & 173 & 20.84000 & $20.84000 \pm 0.002$ \\
\hline
\end{tabular}

for application in conjunction with the miniaturized energy consumption measurement systems employed in clinical NICU environments. The system can simulate the miniscule variations in the respiration of a premature infant, and can be used to establish an appropriate oxygen environment to ensure the well being of the infant. Furthermore, the system can measure various characteristics of the oxygen sensors used to monitor the energy consumption of a premature infant, including the variation in resistance of the oxygen sensor with different oxygen concentrations, the relationship between the sensitivity of the oxygen sensor and the oxygen concentration, and the influence of working temperature and humidity upon the sensitivity of the oxygen sensor.

This study provides three major contributions. Firstly, it has shown that the use of three different supply gases rather than the conventional use of two improves the resolution of the oxygen concentration control from $0.042 \%$ to $0.006 \%$. The proposed system is capable of developing oxygen concentrations in the range of $5 \%$ to $100 \%$. Furthermore, the time required to increase the oxygen concentration from $21 \%$ to $100 \%$ is approximately 5.6 seconds. Secondly, the system supports the automatic simultaneous measurement of all the essential data, including the working temperature of the oxygen sensor, the relative humidity, the resistance of the oxygen sensor, and the oxygen concentrations. Thirdly, this system facilitates the remote monitoring and instructing of the measurement process through its integration with the Internet. Not only does this approach improve the efficiency of the testing process, but it also results in significant reduction of researcher man-hours since the process can be controlled and monitored via remote Internet access rather than requiring the continuous physical presence of a researcher over the extended measurement period. 


\section{ACKNOWLEDGEMENTS}

The current authors gratefully acknowledge the financial support provided to this study by the Ministry of Education in Taiwan under Grant Number EX-91-EFA09-5-4, by the Bioscience Technology Center, National Cheng Kung University, Taiwan and by the Micro-Nano Technology Center in Taiwan.

\section{REFERENCES}

1. Yeh T-F, Lilien LD, Leu S-T and Pildes RS: Increased $\mathrm{O} 2$ consumption and energy loss in premature infants following medical care procedures. Bio Neonate 1984; 46:157-162.

2. Guyton AC and Hall JE: TextBook of Medical Physiology, Ninth Edition. W. B. Saunders Inc, Philadelphia, Pennsylvania, 2000; 889-901.

3. Zoppi G, Luciano A, Cinquetti M, Graziani S and Bolognani M: Respiratory quotient changes in full term newborn infants within 30 hours from birth before start of milk feeding. Eur J Clin Nutr 1998; 52: 360-362.

4. Lin SC, Luo $\mathrm{CH}$ and Yeh T-F: Improve on performance of indirect calorimetry for small preterm infants. Biomed Eng Appl Basi Comm 2001; 13: 109-115.

5. Lin SC, Luo CH and Yeh T-F: A calibrating system of $\mathrm{O} 2$ Consumption and $\mathrm{CO} 2$ production for premature infants. Rev Sci Instrum 2001; 72(3): 1825-1830.

6. Lin SC, Luo $\mathrm{CH}$ and Yeh T-F: Fuzzy oxygen controller for the indirect calorimeter of preterms. $\mathrm{J}$ Med Eng Technol 2001; 25(4): 149-155.

7. Lee G-B, Wu CC, Chen $\mathrm{MH}$ and Luo $\mathrm{CH}$ : Micromachined oxygen gas sensors based on $\mathrm{Li}$ doped tin oxide and micro-heater for miniaturized energy consumption measurement systems. Proceedings of the 1st International Meeting on Microsensors \& Microsystems ( IM $\mu 2$ ), January 12-14, National Cheng Kung University, Tainan, Taiwan 2003: pp. 44.

8. Wu CC, Lee G-B, Luo $\mathrm{CH}$, Chen MH and Hwang WS: Microscopic energy consumption measurement system based on tin oxide thin-film gas sensor and micro-hotplate. Proceedings of Pacific Rim Workshop on Transducers and Micro/Nano Technologies, July 22-24, Chinese Society of Micro-Nano Technology, Xiamen, China 2002: pp. 681-684.

9. Wu CC, Lee G-B, Luo $\mathrm{CH}$ and Hwang WS: Microscopic energy consumption measurement system in use of neonatal growth researches. Proceedings of the 7 th World Congress on
Biosensors, May 15-17, Kyoto, Japan 2002: P36.03.

10. Wu CC, Chen MH, Luo $\mathrm{CH}$ and Lee G-B: Design and fabrication issues on micromachined oxygen sensors for miniaturized energy consumption measurement systems. Sensors Mater 2003; 15-6 (In Press).

11. Guyton AC and Hall JE: Textbook of Medical Physiology, Ninth Edition. W. B. Saunders Inc, Philadelphia, Pennsylvania, 2000; 501-505.

12. Webster JG: Medical instrumentation: application and design, Third Edition. John Wiley \& Sons Inc, United States, 1998; 372-407.

13. Sberveglieri G, Faglia G, Groppelli S, Nelli P and Perego C: Oxygen gas sensing properties of undoped and Li-doped $\mathrm{SnO} 2$ thin films. Sensor Actuat B 1993; 13-14: 117-120.

14. Watson J, Ikohura K and Coles GSV: The tin dioxide gas sensor. Meas Sci Technol 1993; 4: 711719.

15. Lantto V, Romppainen $P$ and Leppavuori S: A study of the temperature dependence of the barrier energy in porous tin dioxide. Sensor Actuat B 1988; 14: 149-163.

16. Göpel W and Schierbaum D: $\mathrm{SnO} 2$ sensors: current status and future prospects. Sensor Actuat B 1995; 26-27: 1-12.

17. Simon I, Bârsan N, Bauer $M$ and Weimar U: Micromachined metal oxide gas sensors: opportunities to improve sensor performance. Sensor Actuat B 2001; 73: 1-26.

18. Solzbacher F, Imawan C, Steffes H, Obermeier E and Möller H: A modular system of SiC-based microhotplates for the application in metal oxide gas sensors. Sensor Actuat B 2000; 64: 95-101.

19. Heilig A, Bârsan N, Weimar U, SchweizerBerberich M, Gardner JW and Göper W: Gas identification by modulating temperatures of SnO2-based thick film sensors. Sensor Actuat B 1997; 43: 45-51.

\section{NOMENCLATURE}

BPS: Bits Per Second

CMOS: Complementary Metal-Oxide Semiconductor. One form of standard Integrated Circuit (IC) cell containing nchannel and p-channel MOSFETs.

DAC: Digital to Analog Converter

DAQ: Data Acquisition. It is used to collect and measure electrical signals from sensors. These data signals are then processed using a computer.

MEMS: Originally MEMS indicated MicroElectro-Mechanical System-microscopic 
mechanical elements. These elements were fabricated on silicon chips using techniques similar to those applied in integrated circuit manufacture and were used as sensors, actuators, and other miniature scale devices. Nowadays, MEMS refers to almost any miniaturized device.

NICU: Newborn Intensive Care Unit.

NRDS: Neonatal Respiratory Distress Syndrome

RS-232C: One form of serial data transfer protocol.

RQ: Respiratory Quotient, which is defined as the ratio of carbon-dioxide generation to oxygen consumption.

SOC: System On a Chip. A novel CMOS-based technology which integrates many devices and circuits into a single chip to form an integrated system. 